\title{
PRODUÇÃo E CARACTERIZAÇÃo FíSICO-QUíMICA DO LICOR DE AÇAí (Euterpe oleracea MART.) OBTIDO COM DIFERENTES FONTES ALCOÓLICAS E TEMPOS DE INFUSÃO
}

\author{
Antonio Maricélio Borges de Souza ${ }^{1 *}$, Edna Jaila Menezes da Silva², Lívia Naiara Silva de Sousa ${ }^{2}$, \\ José Luiz Moraes ${ }^{3}$
}

${ }^{1}$ Universidade Estadual Paulista (UNESP), Campus de Jaboticabal, Jaboticabal, 14884-900, São Paulo, Brasil

${ }^{2}$ Universidade Federal Rural da Amazônia (UFRA), Campus Capitão Poço, Capitão Poço, 68650-000, Pará, Brasil

${ }^{3}$ Universidade Federal Rural da Amazônia (UFRA), Campus Sede, Belém, 66077-830, Pará, Brasil

*Autor para correspondência: Antonio Maricélio Borges de Sousa; e-mail: maricelio_@hotmail.com

Recebido: 2204/2021, Aceito: 1809/2021, Publicado: 18/10/2021

\section{Resumo}

A produção e o consumo do açaí são um forte símbolo de tradição local, e a principal fonte de renda de muitas famílias no Estado do Pará. Objetivou-se com o presente trabalho, elaborar e caracterizar físico-quimicamente o licor à base de açaí cultivar BRS-Pará obtido com diferentes fontes alcoólicas e tempos de infusão. A produção do licor foi realizada de forma artesanal. Os tratamentos foram arranjados em esquema fatorial $2 \times 3$, sendo duas fontes alcoólicas (aguardente de cana-de-açúcar e vodka) e três tempos de infusão (7, 14 e 21 dias). Observou-se que os valores de $\mathrm{pH}$ encontrados para ambas as fontes alcoólicas diminuíram em função do tempo que permaneceram em infusão. O valor médio obtido para sólidos solúveis foi de $21^{\circ}$ Brix. Para acidez total titulável, ambas as fontes alcoólicas alcançaram médias de 1,36 g/100g. Os teores alcoólicos médios foram de 19,44 e 18,75 $\% \mathrm{v} / \mathrm{v}$, respectivamente para aguardente e vodka. As características físico-químicas analisadas do licor elaborado demonstraram ser influenciadas pelos diferentes tempos de infusão e fontes alcoólicas. A produção artesanal do licor de açaí mostrou-se como uma excelente alternativa para a obtenção de bebida de um fruto tipicamente regional, dessa forma, agregando valor ao produto final, visto a facilidade de preparo e aquisição de sua matériaprima, incrementando assim a fonte de renda familiar de pequenos agricultores rurais.

Palavras-chave: Açaí cultivar BRS-Pará, Análises físico-químicas, Bebida alcoólica, Fruta da Amazônia, Inovação tecnológica.

\section{PRODUCTION AND PHYSICAL-CHEMICAL CHARACTERIZATION OF AÇAÍ LIQUOR (Euterpe oleracea Mart.) OBTAINED WITH DIFFERENT ALCOHOLIC SOURCES AND INFUSION TIMES}

\begin{abstract}
The production and consumption of açaí is a strong symbol of local tradition, and the main source of income for many families in the State of Pará. The present study aimed to elaborate and characterize physical-chemically the açaí liquor produced from the cultivar BRS-Pará obtained with different alcoholic sources and infusion times. The liquor production was done manually. The treatments were arranged in a $2 \times 3$ factorial scheme, with two alcoholic sources (sugar cane brandy and vodka) and three infusion times (7, 14 and 21 days). It was observed that the $\mathrm{pH}$ values found for both alcoholic sources decreased as a function of the time they remained in infusion. The average value obtained for soluble solids was $21^{\circ}$ Brix. For total titratable acidity, both alcoholic sources reached averages of $1.36 \mathrm{~g} / 100 \mathrm{~g}$. The average alcoholic levels were 19.44 and $18.75 \% \mathrm{v} / \mathrm{v}$, respectively for brandy and vodka. The physical-chemical characteristics analyzed proved to be influenced by the different infusion times and alcoholic sources. The production of handmade açaí liquor proved to be an excellent alternative for obtaining a drink of a typically regional fruit, thus adding value to the final product, given the ease of preparation and acquisition of its raw material, thus increasing the source of family income for small rural farmers.
\end{abstract}

Keywords: Açaí cultivar BRS-Pará. Alcoholic beverage. Fruit of the Amazon. Physical-chemical analysis. Technological innovation. 


\section{Introdução}

$\mathrm{O}$ açaizeiro (Euterpe oleracea Mart.), de origem nativa da América Central e do Sul, é considerado como a palmeira mais produtiva da região amazônica (Bonomo et al., 2014). Seu fruto, conhecido como açaí, tem forma globosa, de 1 a $2 \mathrm{~cm}$ de diâmetro, e peso médio de 1,5 gramas; dependendo do tipo e da maturação, o epicarpo é roxo ou verde, porém seu consumo não ocorre na forma in natura, necessitando ser processado (Rogez et al., 2011). A produção e o consumo do açaí são um forte símbolo de tradição local, e a principal fonte de renda de muitas famílias no Estado do Pará, correspondendo por aproximadamente $70 \%$ da renda dos extrativistas ribeirinhos (Nogueira et al., 2013).

O açaí constitui a base da alimentação da população paraense, principalmente dos povos ribeirinhos que o consomem frequentemente, seja com farinha de mandioca associado ao peixe, camarão, carne ou outra fonte de proteína que esteja acessível. Isso ocorre, pois, além de apresentar um sabor delicioso, refrescante e acentuado, é uma fruta com valor nutricional altamente energético, contendo alto teor de lipídios, carboidratos, fibras, proteínas, tornando-o um alimento calórico. Além disso, apresenta em sua composição, compostos bioativos, como polifenóis, da classe dos flavonoides, em destaque as antocianinas (Darnet et al., 2011; Kang et al., 2010).

Existem diversas formas de apreciação do açaí, como, por exemplo, nas formas de geleias, polpa congelada, xarope, pó, creme e sorvetes. No mercado brasileiro, o fruto é consumido com finalidade de bebida energética; já no mercado externo, como uma bebida exótica (Darnet et al., 2011; Yamaguchi et al., 2015). Mesmo com toda sua potencialidade, há ainda a necessidade de ampliar cada vez mais essa diversidade de usos, principalmente levando em consideração os produtores de base familiar que não conseguem de alguma forma escoar sua produção e acabam perdendo parte desta por não possuírem tecnologia acessível para armazenagem.

Diante disso, o processamento de frutas torna-se uma boa alternativa para minimizar as perdas póscolheita, principalmente em razão da maioria das frutas tropicais serem altamente perecíveis (Sousa et al., 2010). Aliado a isso, uma variedade de produtos pode ser elaborada para minimizar essas perdas ou reaproveitar resíduos oriundos da agroindústria, entre eles destaca-se o licor de frutas. Dessa maneira a proposta possibilitará o fornecimento de um produto à base do fruto que é símbolo do estado do Pará.

As bebidas alcoólicas ocupam um lugar de destaque nas mais diversas civilizações já existentes (Santos et al., 2018). Os licores são uma categoria de bebidas que tem se reinventado tanto pela evolução tecnológica como pela diversidade de sabores, e obteve um crescimento nas vendas no mercado brasileiro nos últimos anos (Passos et al., 2013). São bebidas que agradam aos paladares mais exigentes, possuindo suas receitas preservadas por gerações, e podem ser servidos tanto em temperatura ambiente como gelados (Marinho et al., 2013; Passos et al., 2013).

As vendas do segmento de licores no Brasil chegam a aproximadamente 7 milhões de litros $\mathrm{ano}^{-1}$. No mundo, os números surpreendem, as cinco maiores marcas deste segmento - entre mais de 300 marcas comerciais de licores existentes - vendem mais de 230 milhões de litros ao ano (Fernandes, 2018). O mercado de licores tem se expandido ao longo dos tempos, por ser uma bebida consumida durante todo ano, não exigindo épocas específicas. Cerca de $20 \%$ da produção de licores no Brasil é destinada à exportação, sendo os licores artesanais mais procurados e também os mais valorizados (Lazzari, 2021).

A elaboração de licores constitui uma forma de solucionar os problemas relacionados à perecibilidade de frutas. Além disso, é outra escolha para enfrentar os problemas relacionados ao excesso de produção e baixos preços praticados em alguns períodos (Teixeira et al., 2010). A fabricação de licor artesanal exige tecnologia simples, o produto final é comercializado à temperatura ambiente e apresenta extensa vida de prateleira (Barros et al., 2008). Com base nessas considerações, o objetivo dessa pesquisa é elaborar e caracterizar físico-quimicamente o licor à base de açaí cultivar BRS-Pará obtido com diferentes fontes alcoólicas e tempos de infusão.

\section{Material e Métodos}

A elaboração do licor e as análises físico-químicas foram realizadas no Laboratório de Química (Multiusuário), da Universidade Federal Rural da Amazônia, Campus de Capitão Poço, Pará, com exceção da análise de teor alcoólico, à qual foi verificada no Laboratório de Processamento e Análises Químicas de Alimentos do Centro de Tecnologia Agropecuária (CTA), da Universidade Federal Rural da Amazônia, Campus Sede, Belém, Pará. A metodologia utilizada para elaboração do licor foi adaptada de Almeida e Gherardi (2019).

O município de Capitão Poço está localizado no Estado do Pará, pertencente à Mesorregião do Nordeste Paraense e na Microrregião Guamá, com as coordenadas geográficas $01^{\circ} 44^{\prime} 54^{\prime \prime}$ de latitude Sul e $47^{\circ} 03^{\prime} 42^{\prime \prime}$ de longitude oeste do Meridiano de Greenwich, distante $226 \mathrm{~km}$ da capital do estado (Oliveira et al., 2016). O clima da região, segundo a classificação de Köppen, é do tipo $A m$ com precipitação 
anual em torno de $2.500 \mathrm{~mm}$, e uma curta estação seca entre setembro e novembro (precipitação mensal em torno de $60 \mathrm{~mm}$ ), temperatura média de $26^{\circ} \mathrm{C}$ e umidade relativa do ar entre 75 à $89 \%$ (Schwart, 2007).

Para a produção do licor foram utilizadas as seguintes matérias-primas: aguardente de cana-deaçúcar com graduação alcoólica $39 \%$ vol. (Pirassununga $\left.{ }^{\circledR}\right)$; vodka roskoff pura tridestilada com graduação alcoólica de 37,5 \% vol. (Roskof®); e açúcar cristal refinado (Carlito $\AA$ ), adquiridos em redes de supermercados; frutos de açaí da cultivar BRS-Pará, obtidos de matrizes cultivadas em uma propriedade rural no município de Capitão Poço, Pará. Os frutos apresentavam no momento da colheita o epicarpo com a tonalidade roxo-escura intensa recoberta por uma camada de pó com a tonalidade branco-acinzentada, caracterizado como sendo o estádio adequado para a colheita destes (Fig. 1).

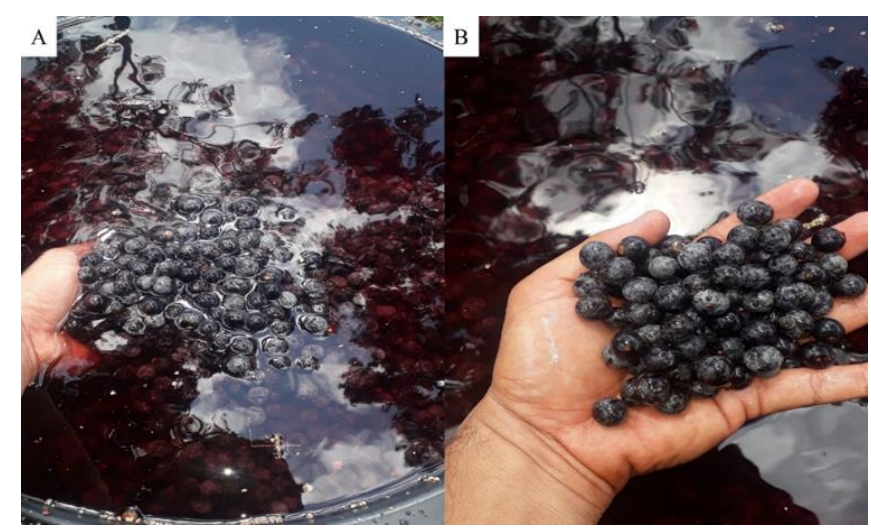

Figura 1. Demonstração do ponto de colheita dos frutos de açaí cultivar BRS-Pará. (A) frutos imersos em água em hiplocorito de sódio para sanitização e (B) frutos após sanitização.

Os licores foram elaborados de forma artesanal adotando as etapas descritas na Fig. 2. Salienta-se que cada passo foi descrito posteriormente de forma concisa, para que haja melhor entendimento a respeito do processo de produção realizado.

No Laboratório de Química (Multiusuário), os frutos foram lavados com água potável corrente, com o intuito de se retirar as impurezas presentes. Após, passaram por uma assepsia por embebição em solução de hipoclorito de sódio (1\%), por 10 minutos, em seguida, foram enxaguados com água corrente e colocados para secar à temperatura ambiente do laboratório.

Posteriormente os frutos foram pesados em balança eletrônica semi-analítica de bancada (Marca BEL®, modelo S622, precisão de $0,01 \mathrm{~g}$ ) e colocados em frascos de vidro com capacidade volumétrica de $500 \mathrm{~mL}$ cada. Ressalta-se que estes foram previamente esterilizados e identificados conforme cada fonte alcoólica e tempo de infusão. A proporção de massa de frutos/volume de fontes alcoólicas $(\mathrm{m} / \mathrm{v})$ foi feita de forma homogênea para todos os tratamentos, sendo 420 $\mathrm{g} / 400 \mathrm{~mL}$, respectivamente. Os recipientes foram fechados hermeticamente e acondicionados em local fresco, arejado e sem luz, respeitando o período de cada tratamento para realização das posteriores etapas.

A etapa de filtração dos extratos alcoólicos foi realizada após cada período de infusão, utilizando uma peneira de nylon de malha fina. Ao final de cada filtragem, obteve-se o volume de $1600 \mathrm{~mL}$ para cada fonte alcoólica e tempos de infusão, sendo reservadas amostras de $500 \mathrm{~mL}$ para realização da análise do teor alcoólico dos extratos.

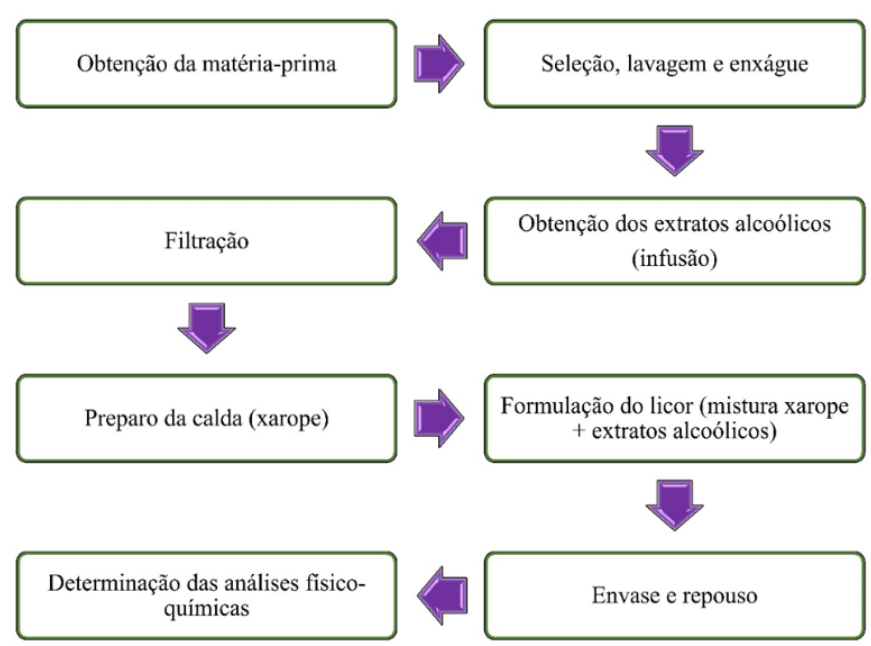

Figura 2. Fluxograma do processo de elaboração do licor de açaí cultivar BRS-Pará produzido com diferentes fontes alcoólicas e tempos de infusão.

Para a preparação da calda (xarope), utilizou-se $1000 \mathrm{~g}$ de açúcar refinado comercial e $1000 \mathrm{~mL}$ de água potável $(\mathrm{m} / \mathrm{v})$. Após resfriamento da mesma em banho de gelo, fez-se a incorporação e homogeneização com $450 \mathrm{~mL}$ de xarope para cada $1100 \mathrm{~mL}$ de extrato alcoólico de cada tempo de infusão. Os licores foram acondicionados em recipientes de vidro com capacidade volumétrica de $2 \mathrm{~L}$ e mantidos em repouso, armazenados em local nas mesmas condições utilizadas no processo de infusão (Tabela 1).

Para avaliar a qualidade dos licores obtidos após as etapas descritas anteriormente, foram utilizadas amostras em triplicatas para determinação das análises físico-químicas: $\mathrm{pH}$; sólidos solúveis totais ( ${ }^{\circ} \mathrm{Brix}$ ); acidez total titulável, de acordo com as normas do Instituto Adolfo Lutz (IAL, 2008); e teor alcoólico, conforme metodologia proposta por Atinks e Jones (2006). 
Tabela 1. Formulação dos licores de açaí cultivar BRSPará produzidos com diferentes fontes alcoólicas e tempos de infusão.

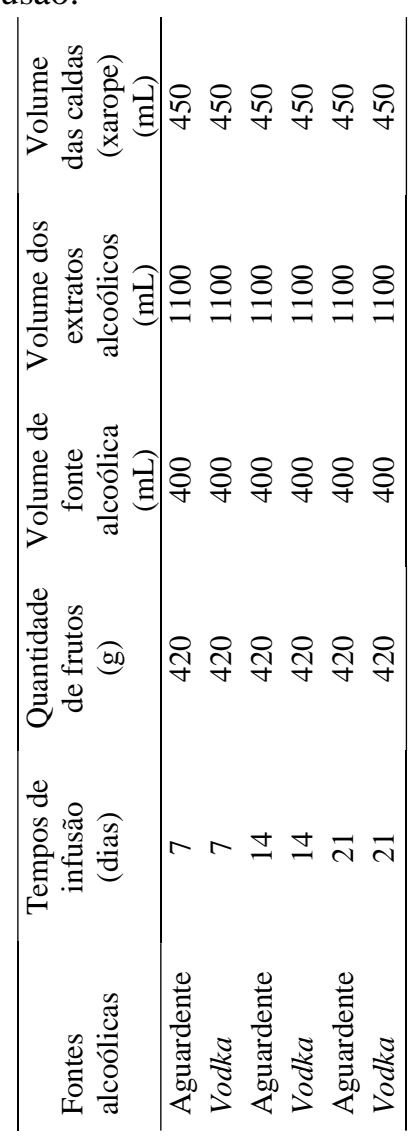

A determinação do $\mathrm{pH}$ foi realizada com a utilização de um pHmetro digital de bancada (Unity®, modelo mPA-210 Bivolt) previamente calibrado em soluçõestampão de $\mathrm{pH}=7$ e 4 (Fig. 3A). Para determinação dos sólidos solúveis totais ${ }^{\circ}$ Brix, foi utilizado refratômetro analógico portátil de leitura direta (Instrutemp ${ }^{\circledR}$, Modelo RHB32, exatidão de $\pm 0.2 \%^{\circ}$ Brix) (Figura 3B). Foram utilizadas quatro gotas como amostra dos licores obtidos para a leitura da percentagem da concentração de açúcar presente nestes. A acidez total titulável foi determinada utilizando o método potenciométrico em razão do licor possuir coloração escura. Utilizou-se como reagente solução de hidróxido de sódio $\mathrm{NaOH}$ 0,1M, usando como indicador a fenolftaleína (Fig. 3C). Posteriormente ao processo de titulação, foi empregada a equação presente da Norma 312IV-IAL (IAL, 2008), para que assim fosse definida a quantidade real de acidez, sendo os resultados expressos em $\mathrm{g} / 100 \mathrm{~g}$ de ácido cítrico. Os teores alcoólicos dos extratos foram obtidos com a utilização do alcoômetro de Gay-Lussac 0/100 ${ }^{\circ}$ Gay-Lussac (Incoterm ${ }^{\circledR}$, modelo 5683) (Fig. 3D).
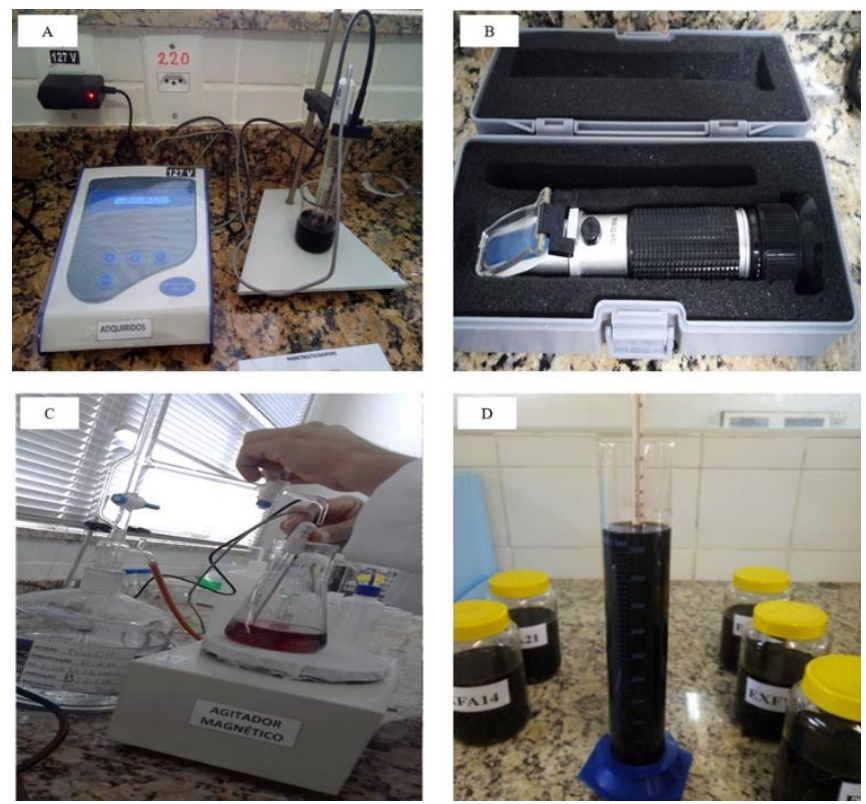

Figura 3. Potenciômetro digital utilizado na medição do pH (A), refratômetro analógico portátil (B), titulação potenciométrica da amostra com solução de hidróxido de sódio (C) e observação direta do teor alcoólico após estabilização do alcoômetro (D).

Para realização do experimento, adotou-se o delineamento experimental inteiramente casualizado. Os tratamentos foram arranjados em esquema fatorial 2 $\times 3$, sendo duas fontes alcoólicas (aguardente de canade-açúcar e vodka) e três tempos de infusão $(7,14$ e 21 dias). Utilizou-se quatro repetições por tratamento, totalizando 24 parcelas. Os dados adquiridos foram demonstrados pelos valores de médias obtidos das triplicatas e plotados em gráficos de barras, para melhor visualização dos resultados. O software utilizado foi o Microsoft Excel® versão 2016.

\section{Resultados e Discussão}

Os produtos prontos são demonstrados na Fig. 4. É possível observar mudanças na coloração do licor, conforme aumento do tempo de infusão, sendo, de coloração mais escura aos 7 dias de infusão (Fig. 4A).

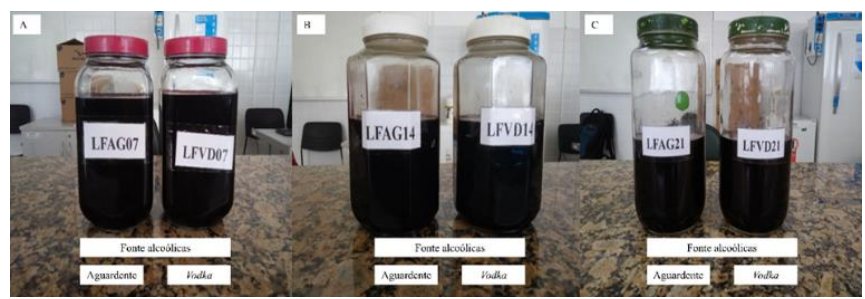

Figura 4. Licores prontos referentes a cada tempo de infusão e fontes alcoólicas. Licores obtidos aos 7 (A), 14 (B) e 21 (C) dias de infusão com suas respectivas fontes alcoólicas. Em que: $\mathrm{LFAG}=$ fonte alcoólica aguardente e LFVD = fonte alcoólica vodka. 
Os valores de $\mathrm{pH}$ encontrados para ambas as fontes alcoólicas diminuíram em função do tempo que permaneceram em infusão, sendo que a vodka obteve médias superiores quando comparadas a aguardente (Fig. 5A), sendo que estes valores estão próximos ao da fruta in natura. Conforme relatado por Cohen et al. (2009), ao determinarem as características físicoquímicas da polpa de açaí da cultivar BRS-Pará encontraram $\mathrm{pH}$ de 4,80 . Isso permite inferir que esta característica é influenciada principalmente pelo período que a fruta fica em contato com a fonte
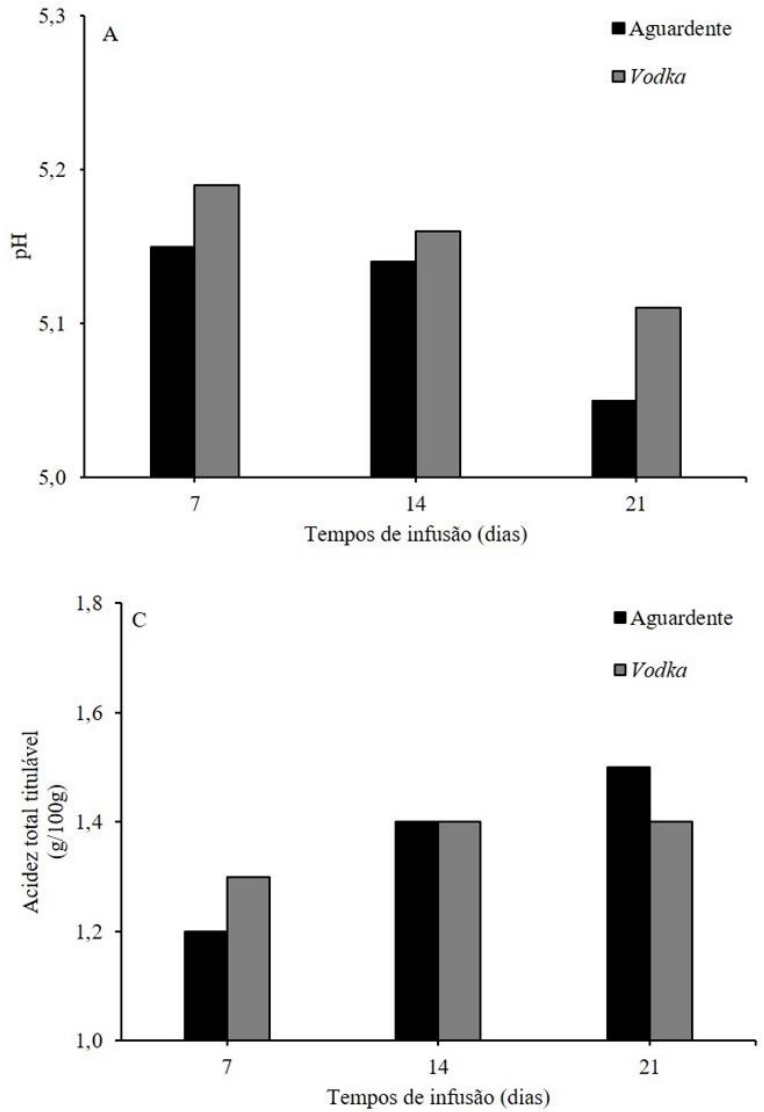

alcoólica durante a elaboração da bebida. Adicionalmente, Teixeira et al. (2012) relatam que os licores que apresentam $\mathrm{pH}$ em torno de 4,5 são significativamente os melhores para conservação, já aqueles com valores mais elevados, são os mais aceitos pelos consumidores. Valores inferiores de $\mathrm{pH}$ ao desta pesquisa foram obtidos por Oliveira, Oliveira de Deus e Caliari (2015) em licores de abacaxi produzidos com diferentes bases alcóolicas e, por Oliveira e Santos (2011) ao elaborarem um licor à base de açaí.
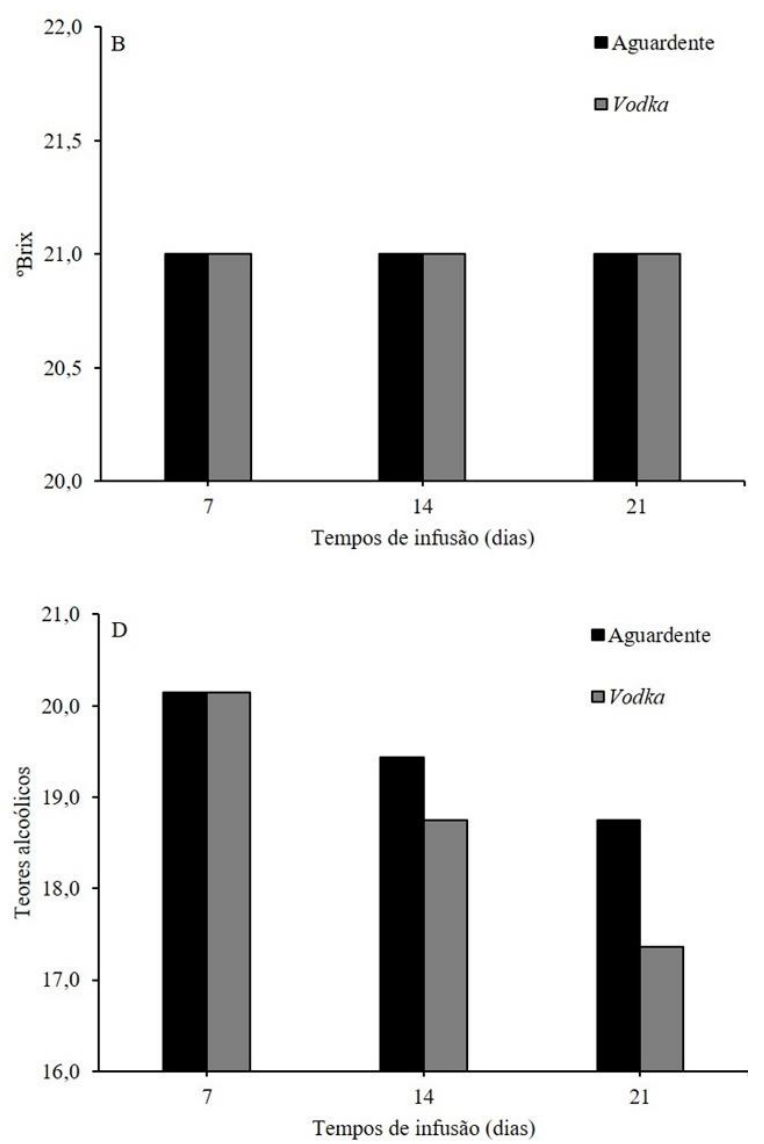

Figura 5. Valores médios das características pH (A), ${ }^{\circ}$ Brix (B), acidez total titulável (C) e teores alcoólicos (D) do licor de açaí cultivar BRS-Pará produzido com diferentes fontes alcoólicas e tempos de infusão.

Observa-se na Fig. 5A e 5C que o tempo de infusão com 21 dias para a fonte alcoólica aguardente apresentou menor e maior valor de média, respectivamente para $\mathrm{pH}$ e acidez total titulável, o que pode favorecer que ocorra o aumento de sua vida de prateleira e uma maior estabilidade dos compostos extraídos pelo processo de infusão utilizado. É importante frisar que o tempo de infusão é um fator importante no processamento de licores, haja vista que se for muito curto não permitirá a completa extração dos componentes desejáveis. No caso das frutas, o tempo em que elas devem permanecer em contato com o álcool varia de acordo com a fruta empregada (Teixeira et al., 2010).
Com relação a característica sólidos solúveis ( ${ }^{\circ}$ Brix), não houve oscilação, sendo o valor médio de 21 ${ }^{\circ}$ Brix obtido para ambas as fontes alcoólicas e tempos de infusão (Fig. 5B). Isto pode ser explicado pelo fato de ter sido adicionada a mesma quantidade de calda de açúcar (xarope) em todos os tratamentos. Além disso, percebe-se que os frutos utilizados se encontravam no mesmo estágio de maturação, em razão da não alteração nos valores. Complementarmente, verificou-que a quantidade de açúcar presente nos licores foi de $290 \mathrm{~g} / \mathrm{L}$ e, segundo Teixeira et al. (2011) o teor de açúcar em licores de frutas varia de 250 a $350 \mathrm{~g} / \mathrm{L}$. Com base nessas informações, o licor obtido é classificado como fino ou doce, pois segundo a Legislação Brasileira de Bebidas vigente essa classificação é destinada a bebida 
que contém mais de 100 e no máximo $350 \mathrm{~g} / \mathrm{L}$ de açúcares (Brasil, 2009). Valor inferior para açúcares totais foi obtido por Oliveira et al. (2019) ao elaborarem licor à base de graviola.

Para acidez total titulável, os resultados demonstram que houve incremento nos teores obtidos conforme se aumentava o período de infusão. A fonte aguardente obteve valores médios de 1,2, 1,4 e 1,5 $\mathrm{g} / 100 \mathrm{~g}$, enquanto que para a vodka foi de $1,3,1,4$ e 1,4 $\mathrm{g} / 100 \mathrm{~g}$, respectivamente para 7,14 e 21 dias. Nota-se que houve estabilização da acidez aos 14 dias para a vodka (Figura 5C). De acordo com Sousa et al. (2013), a acidez é um importante parâmetro na apreciação do estado de conservação de um produto alimentício. Resultados inferiores ao desta pesquisa para acidez titulável foram obtidos por Magalhães et al. (2014), Oliveira et al. (2015) e Almeida e Gherardi (2018) ao desenvolverem licores à base de morango, graviola e goiaba, respectivamente. Essa disparidade está associada ao fato de que cada fruta possui teores específicos de acidez em sua composição química.

Quanto aos teores alcoólicos obtidos no presente trabalho, no tempo de infusão com 7 dias ambas as fontes alcoólicas continham a mesma quantidade em volume de álcool $(20,14 \% \mathrm{v} / \mathrm{v})$, no entanto verificou-se redução destes, conforme aumento do período que passaram em infusão (Figura 5D). Ainda assim, observou-se conformidade dos teores obtidos com os preconizados pela Legislação Brasileira de Bebidas vigente, que prescreve teores de graduação alcoólica na faixa de 15 a $54 \%$ v/v (Brasil, 2009). De acordo com Nunes et al. (2014), as bebidas alcoólicas atingem a esterilidade comercial com aproximadamente $16^{\circ} \mathrm{GL}$ e, a maioria dos licores industriais de frutas possui um teor alcoólico declarado em rótulo entre 18 e $25 \%$ em volume (Teixeira et al., 2007).

Alguns trabalhos junto à literatura reportam teores alcoólicos aproximados ao desta pesquisa, a citar o de Passos et al. (2013), que ao elaborarem licores mistos de cenoura com laranja e com maracujá obtiveram teor alcoólico de $18 \%$ v/v e, Magalhães et al. (2014) em licor de corte de morango com $20 \%$ v/v. Em contrapartida, Oliveira e Santos (2011) ao elaborarem licor de açaí obtiveram valor inferior, sendo sua graduação alcoólica de $16 \%$ v/v e, valores superiores foram alcançados por Silva et al. (2017) ao caracterizarem licores de casca de tangerina, tendo sua graduação entre 28,36 a $34,99 \%$ v/v, sendo os mesmos denominados de licores finos.

É importante ressaltar que, bebidas elaboradas com frutas apresentam em sua composição final, compostos fenólicos provenientes das mesmas e consequentemente poder antioxidante benéfico a saúde, quando o consumo não ultrapassar limites recomendado pela Organização Mundial da Saúde (Letowska et al., 2014). Além disso, o grau de maturação da fruta, a proporção destas e solvente, a concentração da fonte alcoólica e o tempo de extração, podem gerar perfil aromático e gostos diferenciados, influenciando significativamente a qualidade do produto final (Filho et al., 2018). Levando em consideração que os principais atrativos das frutas são cor, aroma, sabor, vitaminas e minerais, deve-se, ao preparar um licor à base de frutas, ter o cuidado de preservar esses atributos e substâncias, de modo que o consumidor possa imediatamente associá-lo à fruta com a qual foi preparado (Penha, 2006).

Considerando que o processamento do licor artesanal exige tecnologia simples, e tem como objetivo principal auxiliar na incrementação de renda do produtor ao enfrentar os problemas relacionados ao excesso de produção e baixos preços praticados em alguns períodos, é de extrema importância que a escolha das matérias-primas seja acessível. Dessa maneira, na elaboração do licor de açaí do presente estudo optou-se por utilizar produtos que são fáceis de se encontrar no mercado local. Os valores dos ingredientes quando a pesquisa foi realizada se encontravam bem abaixo dos atuais, sendo $\mathrm{R} \$ 2,20$ para o açúcar $(\mathrm{Kg}), \mathrm{R}$ \$ 8,00 para a aguardente de cana-de-açúcar (garrafa com $965 \mathrm{~mL}$ ) e $\mathrm{R} \$ 10,00$ para a vodka roskoff (garrafa com $965 \mathrm{~mL}$ ).

\section{Considerações finais}

As características físico-químicas analisadas do licor elaborado demonstraram ser influenciadas pelos diferentes tempos de infusão e fontes alcoólicas. Apesar disso, se encontram dentro dos padrões exigidos pela Legislação Brasileira de Bebidas, sendo este classificado como fino ou doce.

A produção artesanal do licor de açaí mostrou-se como uma excelente alternativa para a obtenção de bebida de um fruto tipicamente regional, dessa forma, agregando valor ao produto final, visto a facilidade de preparo e aquisição de sua matéria-prima, incrementando assim a fonte de renda familiar de pequenos agricultores rurais.

\section{Agradecimentos}

À Universidade Federal Rural da Amazônia, Campus de Capitão Poço, Pará pela formação acadêmica do primeiro e segundo autor.

\section{Referências}

Almeida, J. C.; \& Gherardi, S. R. M. (2018). Elaboração, caracterização físico-química e aceitabilidade de licor de goiaba. Multi-Science Journal, 1:(13), 390-393. 
Almeida, J. C., \& Gherardi, S. R. M. (2019). Elaboração, caracterização físico-química e aceitabilidade de licor de jabuticaba. Revista de Engenharias da Faculdade Salesiana, 10, 20-24.

Atinks, P., \& Jones, L. (2006) Princípios de química: questionando a vida moderna e o meio ambiente $\left(3^{\mathrm{a}}\right.$ ed.). Porto Alegre: Bookman. 965 p.

Barros, J. C., Santos, P. A., Isepon, J. S., Silva, J. W., \& Silva, M. A. P. (2008) Obtenção e avaliação de licor de leite a partir de diferentes fontes alcoólicas. Global Science and Technology, 1:(4), 27-33.

Bonomo, L. F., Silva, D. N., Boasquivis, P. F., Paiva, F. A., Guerra, J. F., Martins, T. A., Torres, Á. G. J., Paula, I. T., Caneschi, W. L., Jacolot, P., Grossin, N., Tessier, F. J., Boulanger, E., Silva, M. E., Pedrosa, M. L., \& Oliveira, R. P. (2014). Açaí (Euterpe oleracea Mart.) modulates oxidative stress resistance in Caenorhabditis elegans by direct and indirect mechanisms. PLoS One, 9:(3), e89933.

Brasil. (2009). Casa Civil. Decreto no ${ }^{\circ} 6.871$, de 4 de junho 2009. Regulamenta a lei $n^{\circ} 8.918$, de 14 de julho de 1994, que dispõe sobre a padronização, classificação, o registro, a inspeção e a fiscalização da produção e do comércio de bebidas. Diário Oficial da União. 33 p.

Cohen, K. O., Mattietto, R. A., Chisté, R. C., \& Oliveira, M. S. P. (2009). Caracterização físicoquímica e funcional da polpa extraída de frutos da cultivar de açaizeiro BRS-Pará. Embrapa Amazônia Oriental (Boletim de pesquisa e desenvolvimento), Belém-PA. 22 p.

Darnet, S., Serra, J. L., Rodrigues, A. M. C., \& Silva, L. H. M. (2011). A high-performance liquid chromatography method to measure tocopherols in assai pulp (Euterpe oleracea). Food Research International, 44:(7), 2107-2111.

Fernandes, C. M. D. (2018). Desenvolvimento $e$ caracterização sensorial de licores de laranja $e$ tangerina. Trabalho de Conclusão de Curso (Bacharelado em Gastronomia) - Departamento de Tecnologia Rural, Universidade Federal Rural de Pernambuco, Recife.

Filho, M. J., Carmo, L. B., Lucia, S. M. D., Saraiva, S. H., Costa, $\quad$ A. $\quad$ V., Osorio, V. M., \& Teixeira, L. J. Q. (2018). Banana Liquor: Optimization of the alcohol and sugar contents, sensory profile and analysis of volatile compounds. LWT-Food Science and Technology, 97, 31-38.
Instituto Adolfo Lutz. (2008). Métodos físico-químicos para análise de alimentos ( $4^{\mathrm{a}}$ ed.). Paulo: Instituto Adolfo Lutz. 1020 p.

Kang, J., Li, Z., Wu, T., Jensen, G. S., Schauss, A. G., $\& \mathrm{Wu}, \mathrm{X}$. (2010). Anti-oxidant capacities of flavonoid compounds isolated from acai pulp (Euterpe oleracea Mart.). Food Chemistry, 122:(3), 610-617.

Lazzari, N. M. Licor, uma bebida consumida independente da estação do ano: Nas quatro estações do ano, o licor é uma bebida muito procurada. Centro de Produções Técnicas e Editora Ltda (c) 2000 - 2021. Acesso em: 14 set. de 2021. Disponível em: <https://www.cpt.com.br/cursos-industriacaseiracomomontar/artigos/licor-umabebida-consumidaindependente-da-estacao-do-ano>.

Letowska, A. S., Kucharska, A. Z., Winska, K., Szumny, A., Nawirskaolszanska, A., \& Mizgier, P. (2014). Composition and antioxidant activity of red fruit liqueurs. Food Chemistry, 157, 533-539.

Magalhães, D. C., Andrade, R. O., Costa, D. P., Santos, D. B., \& Cardoso, R. L. (2014). Desenvolvimento, caracterização físico-química e sensorial de licor de corte de morango. Enciclopédia Biosfera, 10:(18), 1193-1199.

Marinho, S. C., Brito, R. S., Carvalho, M. P., Mouchrek Filho, V. E., Everton, P. C., \& Silva, G. M. (2013). Qualidade físico-química de licores artesanais de jenipapo (Genipa americana L.) comercializados em São Luís, MA. Revista de Higiene Alimentar, 27:(224/225), 78-80.

Nogueira, A. K. M., Santana, A. C., \& Garcia, W. S. (2013). A dinâmica do mercado de açaí fruto no Estado do Pará: de 1994 a 2009. Revista Ceres, 60:(3), 324331.

Nunes, S. P., Teixeira, L. J. Q., Silva, G. B., Bernardes, P. C., \& Rodrigues Júnior, S. (2014). Influência do teor alcoólico na sobrevivência de Staphylococcus aureus em simulados de bebidas alcoólicas. Revista Brasileira de Produtos Agroindustriais, 16:(3), 255-264.

Oliveira, E. N. A., \& Santos, D. C. (2011). Processamento e avaliação da qualidade de licor de açaí (Euterpe oleracea Mart.). Revista do Instituto Adolfo Lutz, 70:(4), 534-541.

Oliveira, E. R., Oliveira de Deus, K., \& Caliari, M. (2015). Production, characterization and acceptability 
of diferente alcohol-based pineapple liqueurs. Revista Verde de Agroecologia e Desenvolvimento Sustentável, 10:(1), 108-114.

Oliveira, F. J., Farias, P. R. S., Silva, A. G., Rodrigues, K. C. V., \& Araujo, F. C. B. (2016). Distribuição espacial da leprose dos citros na Amazônia Oriental. Revista Ciência Agronômica, 47:(1), 56.

Oliveira, E. N. A., Santos, D. C., Santos, Y. M. G., \& Oliveira, F. A. A. (2019). Aproveitamento agroindustrial da graviola (Annona muricata L.) para produção de licores: avaliação sensorial. Journal of Biotechnology and Biodiversity, 7:(2), 281-290.

Penha, E. M. (2006). Licor de frutas. EMBRAPA Coleção Agroindústria Familiar ( $1^{\circ}$ ed.). Brasília. p. 1324.

Rogez, H., Pompeu, D. R., Akwie, S. N. T., \& Larondelle, Y. (2011). Sigmoidal kinetics of anthocyanin accumulation during fruit ripening: a comparison between açai fruits (Euterpe oleracea) and other anthocyanin-rich fruits. Journal of Food Composition and Analysis, 24:(6), 796-800.

Santos, K. M., Machado, M. A., \& Gomes, P. O. M. (2018). Caracterização físico-química, determinação de minerais e avaliação do potencial antioxidante de licores produzidos artesanalmente. Multi-Science Journal, 1:(12), 54-61.

Schwart, G. (2007). Manejo sustentável de florestas secundárias: espécies potenciais no Nordeste do Pará, Brasil. Amazônia: Ciência; Desenvolvimento, 3:(5), 125-147.

Silva, R. C., Amorim, A. B. F., Feitosa, R. M., Oliveira, E. N. A., Feitosa, B. F., \& Amadeu, L. T. S. (2017). Licor fino de casca de tangerina: processamento e caracterização. Arquivos Brasileiros de Alimentação, 2:(3), 164-173.

Sousa, P. H. M., Ramos, A. M., Maia, G. A., Brito, E. S., Garruti, D. S., \& Fonseca, A. V. V. (2010). Adição de extratos de Ginkgo biloba e Panax ginseng em néctares mistos de frutas tropicais. Ciência $e$ Tecnologia de Alimentos, 30:(2), 463-470.

Sousa, F. C., Silva, L. M. M., Castro, D. S., Nunes, J. S., \& Sousa, E. P. (2013). Propriedades Físicas e Físicoquímicas de polpa de Juazeiro. Revista Verde de Agroecologia e Desenvolvimento Sustentável, 8:(2), 6871.
Teixeira, L. J. Q., Ramos, A. M., Chaves, J. B. P., \& Stringheta, P. C. (2007). Testes de aceitabilidade de licores de banana. Revista Brasileira Agrociência, 13:(2), 205-209.

Teixeira, L. J. Q., Rocha, C. T., Junqueira, M. S., Carneiro, J. C. S., \& Saraiva, S. H. (2010). Determinação da cinética de extração alcoólica no processamento de licor de café. Enciclopédia Biosfera, 6:(9), 1-9.

Teixeira, L. J. Q., Simões, L. S., Rocha, C. T., Saraiva, S. H., \& Junqueira, M. S. (2011). Tecnologia, composição e processamento de licores. Enciclopédia Biosfera, 7:(12).

Teixeira, L. J. Q., Simões, L. S., Saraiva, S. H., Junqueira, M. S., \& Sartori, M. A. (2012). Determinação da proporção de açúcar e fruta necessários para conferir os atributos ideais ao licor de abacaxi. Enciclopédia Biosfera, 8:(14), 1883-1889.

Yamaguchi, K. K. L., Pereira, L. F., Lamarão, C. V., Lima, E. S., \& Veiga-Junior, V. F. (2015). Amazon açai: chemistry and biological activities: a review. Food Chemistry, 179, 137-151. 